Miralles Martínez, P., Molina Puche, S. \& Monteagudo Fernández, J. (2017). Cuándo y cómo se evalúa en Geografía e Historia: fases e instrumentos de evaluación del profesorado de ESO. Análisis de entrevistas y grupo de discusión en la Región de Murcia. Revista Electrónica Interuniversitaria de Formación del Profesorado, 20(1), 187-199.

\title{
Cuándo y cómo se evalúa en Geografía e Historia: fases e instrumentos de evaluación del profesorado de ESO. Análisis de entrevistas y grupo de discusión en la Región de Murcia ${ }^{1}$
}

Pedro Miralles Martínez, Sebastián Molina Puche, José Monteagudo Fernández Universidad de Murcia

\section{Resumen}

La evaluación es una parte fundamental del proceso de enseñanza-aprendizaje. Sin embargo, una concepción tradicional de la misma restringe su uso a los resultados de aprendizaje del alumnado, mientras que otra percepción más actual engloba en su campo de actuación, además del aprendizaje discente, la labor docente del profesorado, el propio proceso de enseñanza-aprendizaje y hasta la propia evaluación.

Ambas ideas coexisten a día de hoy en la realidad de las aulas. Para conocer cuál es la más extendida entre el profesorado de Geografía e Historia que imparte docencia en ESO, hemos llevado a cabo un estudio cualitativo, basado en entrevistas y grupo de discusión con catorce docentes, que pretende sacar a la luz las concepciones y las prácticas de evaluación de los profesores en lo relativo a los momentos en los que esta se lleva a cabo y los instrumentos que utilizan para ello. Los resultados nos dicen que, aunque existe diversidad a la hora de entender la evaluación por parte de los docentes, la mayoría se decanta por posicionamientos más actuales que dejan, no obstante, amplio margen de mejora.

Palabras clave:

Evaluación, concepciones del profesorado, fases de la evaluación, Geografía e Historia, ESO

Contacto:

Sebastián Molina Puche, smolina@um.es, Dpto. Didáctica de las Ciencias Matemáticas y Sociales, Facultad de Educación, Universidad de Murcia. Campus de Espinardo, s/n, CP 30100 Murcia.

${ }^{1}$ Este artículo es fruto de los proyectos de investigación "Criterios, procedimientos e instrumentos de evaluación de los contenidos de Geografía e Historia en 2. ${ }^{\circ}$ ciclo de Educación Secundaria Obligatoria" (08668/PHCS/08), financiado por la Fundación Séneca-Agencia de Ciencia y Tecnología de la Región de Murcia en el marco del II PCTRM 2007-2010; y “La evaluación de las competencias y el desarrollo de capacidades cognitivas sobre Historia en Educación Secundaria Obligatoria” (EDU201565621-(3-2-R), subvencionado por el Ministerio de Economía y Competitividad. 


\title{
When and how we evaluate in Geography and History: phases and assessment tools of Secondary Education teachers. Analysis of interviews and discussion group in Murcia (Spain)
}

\begin{abstract}
The assessment is an integral part of the teaching-learning process. However, the traditional conception of assessment restricts its use to the results of student learning. On the contrary, the current perception of assessment includes students learning, the teaching faculty, the process of teaching-learning and even the assessment.

Both ideas coexist today in the classroom. To know which is the most usual among Secondary Education teachers of geography and history, we have carried out a qualitative study, based on interviews and group discussions with fourteen teachers, which aims to show the assessment conceptions and practices of teachers in the relative thing to the moments in which this one is carried out and the instruments that they use for it. The results tell us that, although diversity exists in understanding the assessment by teachers, most of them opted for more current positions that leave, however, wide margin of improvement.
\end{abstract}

\section{Key words}

Assessment, conceptions of the professorship, phases of the assessment, Geography and History, Secondary Education

\section{Introducción}

La evaluación en educación se ha entendido, tradicionalmente, como el proceso por el que se juzgan los resultados del proceso de enseñanza-aprendizaje, en el que se integra, siempre en relación con unos objetivos previstos (Jorba y Sanmartí, 1994).

La concepción más actual de evaluación la considera como un proceso continuo y amplio que afecta a todos los elementos que forman parte del escenario educativo, cuya finalidad no es emitir un juicio de valor sino mejorar el aprendizaje de los alumnos (Hamayan, 1995), el proceso de enseñanza-aprendizaje, la misma docencia y hasta la propia evaluación.Stufflebeam y Shinkfield (1995)identificaban evaluación con perfeccionamiento y mejora que no pretende perfeccionarel aprendizaje actual, sino también el futuro a través de evaluaciones integradoras de todos sus actores, tanto alumnos como docentes, en lo individual y lo colectivo (Crisp, 2012).

Para Clark (2012), las virtudes de la evaluación formativa afloran cuando la información recogida del proceso de evaluación se da a conocer al alumno pues mejora su motivación y su compromiso hacia el aprendizaje, estando más cerca de las escuelas inteligentes de Perkins (1997), donde para enseñar cómo usar el conocimiento se precisaba, entre otras cuestiones, de información clara, práctica y reflexiva. Así, la información 
acumulada tiene un claro fin: ayudar a los alumnos para progresar en su aprendizaje (Villa y Alonso, 1996).

Para alcanzar esa evaluación formativa, que ayuda a la metacognicióny la adquisición de las competencias básicas, es necesario que se utilicen muchos instrumentos de evaluación, distintas técnicas y, además, que se evalúen en las fases inicial, procesual y final. Solo de esta manera se puede conocer qué sabe el alumnado, qué problemas de aprendizaje tiene y cómo solucionarlos.Actuar de otra manera supone tener otra concepción de la evaluación, la tradicional, que tiene como finalidad calificar, enjuiciar y jerarquizar al alumnado.

A la luz de diferentes investigaciones realizadas sobre concepciones y actuaciones de los profesores de Geografía e Historia (en adelante $\mathrm{GeH}$ ), los resultados muestran una línea interesante: frente a una generalizada concepción actual de la evaluación se observan unas prácticas ancladas en los usos tradicionales.

En efecto, cuando se pregunta al profesorado sobre sus concepciones sobre evaluación sus afirmaciones se alejan de la visión tradicional. Así lo demuestran la tesis doctoral de Zaragoza (2003), los estudios de Pérez, Pozo, Pecharromán, Cervi, Martínez y Echeverría (2006), Bautista, Pozo y Municio (2011), Alfageme y Miralles (2014) y Alfageme, Miralles y Monteagudo (2015). En todos ellos las conclusiones son semejantes: el profesorado concede a la evaluación inicial un carácter más pedagógico que administrativo; considera que se ha de emplear gran variedad de instrumentos para evaluar al alumnado; defiende que la evaluación es un proceso de diálogo y mejora tendente a orientar al alumnado;y rechaza los exámenes como única forma de evaluar.

Sin embargo, cuando lo que se analizan son las prácticas de evaluación del profesorado de Secundaria, se observa que estas son mucho más tradicionales que sus propias concepciones. Así lo han demostrado estudios como los de Monteagudo y Villa (2011), que al analizar las programaciones de varios departamentos de GeH de diferentes institutos de la Comunidad Autónoma de la Región de Murcia (CARM) dedujeron que los instrumentos más utilizados por los profesores para evaluar al alumnado eran los exámenes. Unos exámenes que por la tipología de ejercicios sólo servían para lograr la memorización de contenidos de naturaleza conceptual (Monteagudo, Miralles y Villa, 2014) y que difícilmente ayudaban al correcto desarrollo de competencias clave (Miralles, Gómez y Monteagudo, 2012).

De igual modo, el reciente estudio de Monteagudo, Villa y Miralles (2015) sobre la opinión de los inspectores educativos respecto a las prácticas de evaluación de los profesores de $\mathrm{GeH}$ de Secundaria en la CARM mostraba que los docentes realizan la evaluación inicial por meras exigencias del sistema, evaluando al alumnado al final del proceso mediante exámenes, dejando al margen la evaluación continua, confirmando la opinión de Álvarez (2008) y Miralles (2015) de que en la escuela se ha confundido evaluación continua con examen.

El hecho de que existan discordancias entre las creencias del profesorado y sus actuaciones puede deberse a la falta de formación pedagógica y didáctica, a la existencia de presiones sociales y académicas, la necesidad de objetivar la evaluación o su propia situación laboral (Pérez y Carretero, 2009), realidad corroborada por Remesal (2011) al argumentar que en secundaria se siente más la presión de la evaluación como una herramienta de certificación de los logros de los estudiantes, así como la presión de las pruebas tipo PISA.

En todo caso, puede comprobarse a través de las investigaciones expuestas la contradicción entre concepciones y prácticas en evaluación. Para intentar cotejar esos 
resultados, optamos por realizar una serie de entrevistas en profundidad y conformar un grupo de discusión para preguntar al profesorado de GeHcuándo y cómo evaluaban. Sus respuestas han aportado datos claros sobre cuál es, para ellos, la funcionalidad de la evaluación.

Los estudios acerca de las concepciones docentes respecto de la evaluación no son muy numerosos (Bautista, Pozo y Municio, 2011), y conviene continuar escrutando las concepciones del profesorado para conocerlas en profundidad antes de sugerir cambios en las prácticas evaluadoras y para comprobar si las nuevas filosofías centradas en las competencias consiguen una mejora de la calidad educativa (Alfageme y Miralles, 2015).

\section{Metodología}

La finalidad de esta investigación es conocer cómo lleva a cabo el proceso de evaluación el profesorado de GeHde ESO en la CARM mediante la descripción y el análisis de las fases e instrumentos que emplea para tal fin. Para ello nos hemos marcado tres objetivos de investigación:

1. Hacer explícitas las ideas del profesorado de GeHacerca de la evaluación.

2. Averiguar las fases de la evaluación que llevan a cabo en la práctica los docentes de $\mathrm{GeH}$.

3. Identificar las técnicas e instrumentos de evaluación que emplea el profesorado en el aula de Ciencias Sociales.

La realización de esta investigación se ha diseñado siguiendo el procedimiento de un estudio descriptivo mediante entrevistas y grupo de discusión, a través de una metodología cualitativa.

La población objeto de estudio ha sido el profesorado de GeHde la CARM. A través de un muestreo aleatorio simple, debido a la escasa información acerca de las características de la población que poseíamos y la pretensión de que cualquier sujeto tuviese la misma probabilidad de ser seleccionado, se escogieron un total de catorce profesores para participar en la investigación: ocho para la entrevista y seis para el grupo de discusión.

Para una mayor información acerca de las características de la muestra y de los instrumentos de recogida de información nos remitimos a Monteagudo, Molina y Miralles (2015).

\section{Análisis y discusión}

EL grupo de discusión surgió como un complemento a las entrevistas realizadas a los profesores,a fin de cotejar los resultados obtenidos de ambas pruebas,por lo que las categorías son idénticas en ambos casos.

Tras la transcripción de la grabación del grupo de discusión mediante el programa Dragon y el análisis de contenido para poder identificar categorías o referentes en base a los cuales realizar el estudio de las diferentes intervenciones de los participantes en torno a la evaluación, obtuvimos un total de tres categorías y ocho subcategorías que resumimos en la tabla 1. 
Tabla 1.

Categorías halladas en las entrevistas y grupo de discusión

\begin{tabular}{|c|c|c|}
\hline \multirow{5}{*}{$\begin{array}{l}\text { 1. Evaluaciones } \\
\text { Cómo se desarrolla la } \\
\text { evaluación a lo largo } \\
\text { del curso }\end{array}$} & \multicolumn{2}{|c|}{$\begin{array}{l}\text { 1.1. Diagnóstica } \\
\text { Se realiza una evaluación diagnóstica y de qué manera }\end{array}$} \\
\hline & \multicolumn{2}{|c|}{$\begin{array}{l}\text { 1.2. Inicial } \\
\text { Se realiza una evaluación inicial y de qué manera }\end{array}$} \\
\hline & \multicolumn{2}{|c|}{$\begin{array}{l}\text { 1.3. Procesual } \\
\text { Se realiza una evaluación continua y de qué manera }\end{array}$} \\
\hline & \multicolumn{2}{|c|}{$\begin{array}{l}\text { 1.4. Final } \\
\text { Se realiza una evaluación final y de qué manera }\end{array}$} \\
\hline & \multicolumn{2}{|c|}{$\begin{array}{l}\text { 1.5. Fase prioritaria } \\
\text { La fase más prioritaria a la hora de la evaluación }\end{array}$} \\
\hline \multirow{8}{*}{$\begin{array}{l}\text { 2. Instrumentos de } \\
\text { evaluación y } \\
\text { calificación } \\
\text { Cuáles son los } \\
\text { instrumentos que se } \\
\text { utilizan para evaluar } \\
\text { al alumnado y cómo } \\
\text { se califican }\end{array}$} & \multirow[t]{7}{*}{$\begin{array}{l}\text { 2.3. Exámenes } \\
\text { El examen como } \\
\text { instrumento de evaluación }\end{array}$} & $\begin{array}{l}\text { 2.3.1. Periodicidad } \\
\text { Cada cuánto tiempo se hacen } \\
\text { exámenes }\end{array}$ \\
\hline & & $\begin{array}{l}\text { 2.3.2. Preguntas } \\
\text { Qué tipo de cuestiones se } \\
\text { preguntan en el examen y si hay } \\
\text { cuestiones opcionales }\end{array}$ \\
\hline & & $\begin{array}{l}\text { 2.3.3. Porcentaje } \\
\text { Qué porcentaje de la nota se } \\
\text { concede al examen }\end{array}$ \\
\hline & & $\begin{array}{l}\text { 2.3.4. Puntuación } \\
\text { En el examen se pone la } \\
\text { puntuación correspondiente a } \\
\text { cada pregunta }\end{array}$ \\
\hline & & $\begin{array}{l}\text { 2.3.5. Ventajas } \\
\text { Ventajas de basar la evaluación } \\
\text { en los exámenes }\end{array}$ \\
\hline & & $\begin{array}{l}\text { 2.3.6. Inconvenientes } \\
\text { Inconvenientes de basar la } \\
\text { evaluación en los exámenes }\end{array}$ \\
\hline & & $\begin{array}{l}\text { 2.3.6. Ausencia } \\
\text { Se puede evaluar sin examen }\end{array}$ \\
\hline & \multicolumn{2}{|c|}{$\begin{array}{l}\text { 2.3. Otros instrumentos } \\
\text { Otros instrumentos que se utilizan en la evaluación }\end{array}$} \\
\hline $\begin{array}{l}\text { 3. Corrección } \\
\text { Cómo hace la } \\
\text { corrección de } \\
\text { exámenes, trabajos el } \\
\text { docente a lo largo de } \\
\text { la evaluación }\end{array}$ & \multicolumn{2}{|c|}{$\begin{array}{l}\text { 3.1. Entrega de exámenes } \\
\text { Se entregan los exámenes a los alumnos }\end{array}$} \\
\hline
\end{tabular}

A continuación, junto al análisis de los resultados por categorías, mostramos diferentes ejemplos de las aportaciones obtenidas de los profesores entrevistados y de los docentes que participaron en el grupo de discusión sobre cada una de las categorías y subcategorías 
halladas. Las hemos agrupado por subcategorías, seleccionando los ejemplos más representativos de entre las respuestas dadas.

Primera categoría: fases de la evaluación o los diferentes momentos en los que esta se desarrolla a lo largo del proceso de enseñanza-aprendizaje. En este caso encontramos cinco subcategorías. La primera subcategoríaestá relacionada con la evaluación diagnóstica que se realiza a inicios de curso. Aquí las respuestas son variopintas, pero la mayoría destaca que lo que se realiza en las aulas es un "un control de contenidos puros y duros tradicional, y ya está" $\left(\mathrm{P}_{2}\right)$, "[... ] simplemente se hace un cuestionario un poco de lo que se va a dar" (E2). Tal y como argumentaba otro participante, “[...] somos más tradicionales. Confeccionamos una prueba inicial para todo el Departamento, eso sí, que procuramos que sea la misma para todo el nivel [... ] pero no hacemos más" (P3). Como puede comprobarse, la evaluación diagnóstica está relacionada con la concepción tradicional de la evaluación, centrada en un examen escrito para comprobar el nivel de conocimientos de los alumnos. No se obtiene ninguna otra información ni se le concede otro valor, como llegan a reconocer abiertamente: "a la inicial nada, no le hago caso. Más que nada es por si viene el inspector que tenga ahí el papel..." (E2). Para una parte del profesorado la evaluación diagnóstica se realiza por meras exigencias administrativas, carece de valor real. Sin embargo, la realidad de los centros educativos no es uniforme y destacan docentes que la hacen para "saber la partida del alumno, pero no una evaluación inicial sobre conocimientos solo, sino una evaluación inicial sobre valores del propio alumno, sobre normas de trabajo del propio alumno, sobre cómo él ha trabajado, cómo le gustaría trabajar [...] Utilizo dos instrumentos: uno oral, porque les voy haciendo preguntas a los alumnos sobre sus antecedentes, cómo han estado, de qué centros proceden, cuál es su situación social, familiar [... ] Luego también una parte escrita, no preguntas concretas, sino para ver la capacidad de redacción, de expresión y la capacidad de relacionar; una pequeñísima parte que son aspectos muy concretos para ver la memoria, una parte de memoria que tienen sobre datos concretos" (E8).

Otra vía por la que apuestan otros profesores es la de "estar experimentando la evaluación diagnóstica a través de trabajos colectivos, para retroalimentar el recuerdo" $(\mathrm{P} 1)$.

La segunda subcategoría encontrada es la que se refiere a la evaluación inicial que tiene lugar al principio de cada unidad didáctica. Hay docentes que mantienen las pruebas escritas de la evaluación diagnóstica, mediante "una batería de preguntas escritas" (E1), pero la mayoría se decanta por otros métodos, como "hojear el tema, que miren las fotos, que vean si les recuerda algo, esto qué significa, vosotros qué creéis" ( $\left.\mathrm{P}_{2}\right)$, o bien, para detectar los conocimientos previos del alumnado, "una especie de lluvia de ideas. Con esa lluvia de ideas voy elaborando una especie de esquema o mapa en la pizarra" (P5).

Aun así, hay profesores que no realizan evaluación inicial alguna "porque la evaluación inicial se mezcla con la procesual, se puede hacer a lo largo del curso, vas conociendo los conocimientos del alumno y su progreso a lo largo del curso: [...] con lo cual no hago ninguna prueba objetiva" (E6).

Así pues, en relación a la evaluación de diagnóstico a inicios de curso para comprobar los conocimientos de los alumnos, observamos que esta suele realizarse como mero trámite burocrático sin valor pedagógico, aunque hay casos en que se realizan varias sesiones recordatorias.

La realidad descrita coincide en parte con las conclusiones presentadas por Buendía, González y Carmona (1999), cuando comprobaron que la mayoría de profesores de GeH empleaban exámenes y pruebas objetivas para hacer aflorar los conocimientos previos del alumnado, y que para la mayoría de docentes era la única vez que realizaban una evaluación 
inicial. Es la falta de formación del profesorado en lo que a la evaluación concierne lo que lleva a mantener las mismas prácticas durante tanto tiempo (Monteagudo, Molina y Miralles, 2015).

No obstante, la evaluación inicial es la que más carencias presenta porque no se hace correctamente y se confunde con la evaluación diagnóstica (Alfageme, Miralles y Monteagudo, 2015).

La tercera subcategoría está relacionada con la evaluación procesual. Aquí aparecen dos conjuntos de docentes bien diferenciados en cuanto a los instrumentos que emplean. Por un lado, encontramos unos profesores que utilizan "la revisión del cuaderno del alumno, que es casi constante" (P1), aquellos quienes le están "dando mucha importancia (... ) a usar el comentario de texto como forma de evaluar paralela" (P4); y quienes emplean los "trabajos en clase en grupo, trabajos individuales, acceso a las TIC" (E5).

Frente a este grupo se posicionan los que afirman que "para mí es el trabajo de los exámenes, del día a día, de toda semana, ya te digo que yo les hago muchos exámenes por evaluación" (E2), y aquellos que defienden abiertamente que "la procesual, hacemos dos exámenes cada trimestre, a parte también mandamos algunos trabajos que son voluntarios, que suben nota también, y eso es lo fundamental" (E4).Es este segundo grupo de profesores el que identifica evaluación con examen (Heredia, 2002) y parece ser el mayoritario dentro de las aulas (Alfageme, Miralles y Monteagudo, 2015; Monteagudo, Villa y Miralles, 2015). Una situación que no parece haber cambiado desde que Buendía, González y Carmona (1999) hallaran en su estudio que el 40\% de los profesores de $\mathrm{GeH}$ realizan exámenes después de cada unidad didáctica.

En lo relativo a técnicas e instrumentos, diferentes al examen, empleados en la evaluación continua, llama la atención el poco empleo de la autoevaluación. Aunque ayuda a la autorregulación del trabajo y el aprendizaje del estudiante, las actividades de autoevaluación son muy escasas en las aulas (Monteagudo, Villa y Miralles, 2015).

La siguiente subcategoría hace referencia a la evaluación final que se realiza al término de cada tema o al fin de cada trimestre o curso. En este aspecto la posición de los docentes es casi unánime: "un examen final y siempre van uno o dos temas del temario del trimestre" (E3) en el que "le volvemos a meter toda la materia, [... ] en realidad el último examen global es para todos el mismo" (E7). Para el profesorado encuestado, la evaluación final o sumativa sería "un examen que puede ser un examen final de trimestre o final de evaluación, como queramos llamarle, donde trato de recoger todo lo que se ha visto durante todo el proceso" (E9). Esta situación concuerda nuevamente con la descrita por Buendía, González y Carmona (1999).

Hay docentes para quienes ese examen final no han de hacerlo todos los alumnos, sino que "solo van los que les quede ya o los que quieran subir nota" (E1), es decir, "los alumnos que no han ido aprobando. Si el alumno ha aprobado la primera, segunda y tercera evaluación lo que le hago es la media, no evaluación continua" (E2). De este modo "la evaluación final sería de las notas que he sacado de las pruebas objetivas que se han hecho, le añadimos pues el porcentaje actitudinal" (E5).

A tenor de estas declaraciones queda acreditado que la evaluación final es, para muchos de ellos, el último examen del trimestre o del curso al que tiene que someterse todo el alumnado o aquellos que tienen una parte de la materia suspensa o quieren subir nota. Para otros la evaluación final es la media de las calificaciones de los diferentes exámenes o de los anteriores trimestres, difiriendo, en este punto, del estudio de Alfageme, Miralles y Monteagudo(2015) en el que los datos indicaban que para los docentes la evaluación final no es un promedio de los resultados de las evaluaciones parciales 
realizadas a lo largo del curso, cuestión que achacaban al propio carácter de la asignatura impartida.

La última subcategoría indica que la inmensa mayoría de participantes en el estudio apuntan a la procesual como la fase más importante de la evaluación pues "es la que haces a lo largo del curso" (E2). Pero no faltan quienes otorgan la misma importancia a todas las fases del proceso, pues "cada una tiene su importancia en la medida que nos sirve para investigar sobre el proceso de enseñanza-aprendizaje" (E5).

A pesar de estas afirmaciones, la confusión de evaluación con examen hace que la realización de exámenes continuos sea la parte más significativa del proceso de enseñanzaaprendizaje en el aula, por lo que no se da una auténtica evaluación continua y sí evaluación final representada en los exámenes, como expresan los inspectores educativos (Monteagudo, Villa y Miralles, 2015).

La segunda gran categoría encontrada en este estudio es la relacionada con los instrumentos de evaluación que emplean en las diferentes fases descritas. La subcategoría más importante vuelve a tener que ver con el examen, y más concretamente con la frecuencia con que estos se realizan. Las respuestas de los profesores se sitúan entre dos opciones, quienes realizan "uno por evaluación [...] hay momentos en que son dos" (E1), y quienes hacen "un examen de cada tema, les hago un ejercicio de cada tema" (E8). Si seguimos esta última opción, vemos que cada "tema suele durar dos semanas" (E5), y que a lo largo del trimestre "hemos dado tres o cuatro temas" (E5), encontramos que hay grupos de alumnos que han de hacer tres o cuatro exámenes en un trimestre. En palabras de Álvarez (2008), en las aulas se examina mucho y se evalúa poco.

La segunda subcategoría sobre instrumentos de evaluación trata del tipo de cuestiones que se preguntan en el examen. Las respuestas de los docentes coinciden en que todos utilizan ejercicios de desarrollo: "Hombre, yo no hago exámenes tipo test. Son exámenes de desarrollo." (E2). Hay incluso quien diferencia por cursos: "La pregunta más larga que podemos poner para tercero de la ESO puede ocupar medio folio, que son muy dirigidas. Para cuarto sí son más largas" (E3). Otras actividades son "preguntas de conceptos, de definiciones" (E1), "y de vocabulario" (E6). También, dependiendo del curso, introducen "una parte que siempre es de procedimientos dependen de la materia que se esté dando" (E7), ya que "cuando estamos en Geografía, siempre les ponemos algún mapa o algún gráfico" (E4) y "en cuarto son más bien comentarios (...) en el tema del arte sí que se les pueden poner imágenes" (E3). En estos exámenes la mayoría de profesores ofrecen optatividad a sus alumnos en ciertos ejercicios, como "en las preguntas más largas o de desarrollo" (E4), contrastando en este punto con la investigación de Monteagudo, Miralles y Villa (2014).

La tercera subcategoría sobre los exámenes trata el porcentaje de la nota final del alumnado que tienen los exámenes. En todos los casos se sitúa por encima del 50\% de la calificación, situándose la mayoría entre "un 60\% aproximadamente" (E9) y "el 80\% de la nota" (E1). Se confirma, pues, que el examen es el instrumento más importante para calificar al alumnado.

La cuarta subcategoría aborda el hecho de si en los exámenes aparece la puntuación de cada ejercicio. Casi todos los docentes afirman que "sí. En cada pregunta se pone entre paréntesis la puntuación" (E1). Aunque quienes no anuncian la puntuación de cada ejercicio admiten que es debido a que "siempre pongo los exámenes deprisa y corriendo y entonces ellos me preguntan lo que valen y yo les digo que lo apunten" (E2).

La siguiente subcategoría informa de las ventajas que tiene para los docentes basar la evaluación en los exámenes. En este punto los docentes aseguran que la principal ventaja 
se encuentra en "la objetividad [... ] porque tienes un material que se lo puedes enseñar a los padres y a los mismos críos [... ] y en esto es en lo que nos basamos" (E4). La búsqueda de amparo en la objetividad hace buena la afirmación de Álvarez (2010) de que el resultado de las evaluaciones se transforma en una nota que "objetiva" la calidad de la respuesta. De esta forma llega a identificarse inteligencia con calificaciones.

Otros argumentos esgrimidos son su utilidad como "recapitulación de todos los contenidos explicados y todas las prácticas realizadas" (E1), y que el elevado número de alumnos en las aulas dificulta otros instrumentos más cualitativos, ya que "cuando tienes un grupo de segundo de Bachillerato con 38 alumnos [...] es mucho más sencillo hacer un examen y curarte en salud" (P5).

Por el contrario, la subcategoría hallada a continuación desarrolla los inconvenientes que tiene para los docentes basar la evaluación en los exámenes. Entre ellos se encuentra que esta forma de evaluar "no es la más justa" (P5) porque "premia a los alumnos que son más académicos" (E4). En este punto parecen coincidir con los inspectores educativos, para quienes cimentar la evaluación en exámenes tiene como consecuencia negativa el hecho de favorecer, generalmente, a los alumnos de estable situación económico-social (Monteagudo, Villa y Miralles, 2015). En este sentido, cabe preguntarse si la escuela sigue actuando como filtro que elimina a los más desfavorecidos y reproduce la estructura social existente.

A ello se suman los profesores que argumentan que basar la evaluación en exámenes supone que los alumnos solamente se esfuerzan por aprobar estos, ya que al resto de elementos no se le da importancia: "Si en los criterios de calificación el $90 \%$ es nota del examen, y estamos haciendo exámenes continuamente, ¿cómo queremos que tenga valor un trabajo?" $(\mathrm{P} 2)$.

Para concluir la categoría de los instrumentos de evaluación, haremos referencia a la segunda gran subcategoría obtenida, entroncada con otros instrumentos que emplean los docentes para evaluar al alumnado. Insisten todos ellos en el cuaderno de los discentes como instrumento de evaluación, cuaderno que "tienen que hacer todos con las actividades que mandamos por evaluación" (E4). Junto a él, otro elemento son los "trabajos individuales o en grupo" (E5), unos trabajos grupales en los que "valoro si colaboran o no, procuro hacer los trabajos de tal manera que todos los alumnos se tengan que implicar, el método cooperativo" (E5).

Un tercer elemento lo constituyen las preguntas o ejercicios de clase que "son corregidos en clase y luego son evaluados" (E8) a través de "un registro de observación de tareas" (P1).

Por último, varios profesores también realizan "exámenes de lectura [...] los críos se leen tres libros o dos libros al año y eso también entra dentro de la nota de la evaluación" (E2), ya que para ellos es muy importante "que el alumno lea y comprenda lo que lee" (E3). Solamente un docente hace referencia a la autoevaluación y otro a la coevaluación, procedimientos que favorecen la evaluación formativa y están en la línea de las nuevas concepciones sobre evaluación.

La última categoría guarda relación con la corrección de los exámenes, si se entregan corregidos a los alumnos. La mayoría de docentes de nuestro estudio sí entrega los exámenes corregidos al alumnado, ya que "además es obligación del profesor entregar los exámenes y enseñárselos a los padres, si quieren, y demás" (E5). La forma de proceder de los docentes en este sentido es la de "repartir [el examen] a cada uno, lo consultan y luego corregimos todos juntos los errores y luego, una vez que ya he corregido con ellos, ya sea 
que tienen alguna duda concreta de por qué me has evaluado aquí o allá" (P5). Hay quienes incluso "además se los doy para que lo firme el padre. Ellos se los llevan a casa..." (E4).

Los profesores que llevan a cabo esta tarea están cerca de lo que preconiza Álvarez (2008) para que el examen cumpla funciones educativas válidas que tienen que ver con la evaluación formativa: permitir participar en la corrección de los exámenes a los alumnos para que sean conscientes de sus logros y lagunas.

Sin embargo, los propios profesores son conscientes de que la situación descrita "no es un hecho habitual en Educación Secundaria: ni es habitual devolver los exámenes a los alumnos corregidos ni es habitual corregirlos en clase. Yo creo que es un problema del sistema" (E8). Así pues, por las aportaciones de nuestros docentes sabemos que son mayoría quienes no entregan los exámenes corregidos al alumnado porque, como indicaba uno de los participantes, "como ya no me fío y el enseñarles los exámenes les sirve para que comparen lo que le has puesto a uno y lo que le has puesto a otro, no para ver ellos sus errores, entonces como no me da la gana, no se los enseño nunca; bueno sí se los enseño, pero no a todos en clase en común. Lo que pasa que nunca vienen a verlos, nunca, porque es en el recreo. Nunca" (E2).

\section{Conclusiones e implicaciones educativas}

Una vez expuestos y comentados los resultados de nuestra investigación, estamos en disposición de relatar las siguientes conclusiones:

- Por lo que a las fases de la evaluación respecta, los profesores de GeH afirman realizar todas las fases de la evaluación, pero en lo que a la evaluación diagnóstica concierne, la forma de proceder es mediante exámenes escritos que tienen como fin comprobar el nivel de conocimientos del alumnado sobre la materia. Algunos de los profesores encuestados afirman abiertamente que esta fase la realizan por exigencias del sistema, no por convencimento.

- En relación a la evaluación procesual, parte importante de los profesores basa dicha evaluación en exámenes escritos de contenidos, mientras que otra parte asegura emplear otros instrumentos (cuaderno del alumno, trabajos escritos, registros de tareas). Verificamos que en el aula se sigue confundiendo evaluación continua con examen continuo, y que, a pesar de emplear otros instrumentos en esta fase de la evaluación, el examen sigue siendo la herramienta de calificación con más peso.

- El examen es el instrumento empleado por estos docentes para llevar a cabo la evaluación final.

- Los profesoresencuestadosaseveran que la evaluación procesual es la más importante, sin embargo el hecho de realizarla en base a exámenes hace que se confunda evaluación continua con examen continuo.

- Los ejercicios que suelen aparecer en los exámenes de GeHsuelen ser temas a desarrollar, seguidos de definiciones de conceptos y vocabulario del tema. Este tipo de ejercicios pone en juego la capacidad de recuerdo del alumnado (Monteagudo, Miralles y Villa, 2014), lo que, unido a la escasa presencia de ejercicios de aplicación de procedimientos dota a la enseñanza de las ciencias sociales de un perfil memorístico basado en elementos conceptuales.

- La mayor ventaja que encuentran los enseñantes de GeH en basar la evaluación del alumnado en los exámenes es el supuesto carácter objetivo que presenta el examen escrito ante posibles reclamaciones, además de suponer una recapitulación de todos los 
contenidos tratados en el aula y un instrumento eficaz ante las elevadas ratios de las aulas. Otras investigaciones (Sainz, 2002), pusieron de manifiesto que la insuficiente formación de los docentes suponía un escollo para emplear técnicas e instrumentos de evaluación cualitativa.

- Sin embargo, son conscientes de que basar la evaluación en exámenes es injusto, ya que benefician más a un tipo determinado de alumnado.

- Los docentes que han colaborado en nuestro estudio afirmaban que devolver corregidos los exámenes al alumnado no es una práctica habitual en las aulas, por lo que no se lleva a cabo una auténtica evaluación formativa, aquella que ayuda a aprender y forma a los estudiantes (Álvarez, 2008).

Con el fin de mejorar el proceso de evaluación en las aulas de Ciencias Sociales de ESO y superar algunos de los obstáculos encontrados en nuestra indagación, lanzamos una serie de propuestas que estén en consonancia con las reformas y avances acaecidos en los últimos tiempos en el mundo educativo. Primeramente, apostamos por la mejora en la formación docente, tanto inicial como continua, para inculcar en los docentes una nueva concepción de la evaluación como proceso, como herramienta formativa y de aprendizaje que emplee una mayor variedad de instrumentos de evaluación además de los tradicionales exámenes, que han de dejar de ser el principal recurso para calificar a los alumnos. Nos referimos al uso de técnicas e instrumentos de carácter cualitativo que permitan una mayor autonomía y formación de los estudiantes, tales como los métodos de indagación, los portafolios, las rúbricas o los cuestionarios de autoevaluación y coevaluación. Todo ello posibilita comprobar la adquisición de conocimientos y las competencias, al tiempo que ayuda a la autorregulación de los aprendizajes y proporcionan mayor interacción entre docentes y discentes (Alfageme y Miralles, 2009). Por último, sin renunciar al examen como instrumento de evaluación, nuestra propuesta va encaminada a modificar los propios exámenes como herramienta evaluativa. Con el fin de abandonar el perfil memorístico que presentan al estar basados en ejercicios de desarrollo de temas, estos se han de elaborar en torno a ejercicios más complejos que pongan en juego aspectos procedimentales de aplicación, más relacionados con las competencias básicas, aumentando para ello la presencia de comentarios de texto, imágenes, mapas, etc., así como ejercicios de valoración o evaluación que también demanden la puesta en juego de elementos actitudinales. También sería necesario modificar su peso cara a la evaluación final para que sea visto como otra actividad de evaluación y oportunidad de aprendizaje, y racionalizar así su uso, en el sentido de que pierdan presencia a favor de otras maneras de evaluar y pasar de un examen continuo a una verdadera evaluación continua.

\section{Referencias}

Alfageme, M. ${ }^{a}$ B. y Miralles, P. (2009). Instrumentos de evaluación para centrar nuestra enseñanza en el aprendizaje de los estudiantes. Íber. Didáctica de las Ciencias Sociales, Geografía e Historia, 60, 8-20.

Alfageme, M. B. y Miralles, P. (2014). El profesorado de Geografía e Historia de Enseñanza Secundaria ante la evaluación. Educar Em Revista, 52, 193-209.

Alfageme, M. ${ }^{a}$ B., Miralles, P. y Monteagudo, J. (2015). Cómo evalúa el profesorado de Geografía e Historia de Enseñanza Secundaria. Revista Complutense de Educación 26 (3), 571-589.

Álvarez, J. M. (2008). Evaluar para conocer, examinar para excluir. Madrid: Morata. 
Álvarez, J. M. (2010). El curriculum como marco de referencia para la evaluación educativa. En J. Gimeno Sacristán, Saberes e incertidumbres sobre el currículum (pp. 355-371). Madrid: Morata.

Bautista Arellano, A., Pérez Echevarría, M. ${ }^{a}$ y Pozo Municio, J. (2011). Concepciones de profesores de piano sobre la evaluación. Revista de Educación, 355, 443-466.

Buendía, L., González, D. y Carmona, M. (1999). Procedimientos e instrumentos de evaluación en Educación Secundaria. Revista de Investigación Educativa, 17(1), 215236.

Clark, I. (2012). Formative Assessment: Assessment Is for Self-Regulated Learning. Educational Psychology Review, 24 (2), 205-249.

Crisp, G. T. (2012). Integrative Assessment: Reframing Assessment Practice for Current and Future Learning. Assessment \& Evaluation in Higher Education, 37 (1), 33-43.

Hamayan, V. E. (1995). Approaches to Alternative Assessment.Annual Review of Applied Linguistics, 15, 214-219.

Heredia, A. (2002). El conocimiento práctico de la evaluación en los profesores. Zaragoza: Prensas Universitarias.

Jorba J. y Sanmartí, N. (1994). Las redes sistémicas. Enseñar, Aprender y Evaluar. Un proceso de Evaluación Continua. Barcelona: Ministerio de Educación y Cultura.

Miralles, P. (2015). Investigaciones sobre evaluación en ciencias sociales en la educación básica. Propuestas para la mejora de la práctica evaluativa. En Didáctica de la historia. Problemas y métodos (II, pp. 99-129). México: Editorial y Servicios Culturales El Dragón Rojo.

Miralles, P., Gómez C. J. y Monteagudo, J. (2012). La evaluación de la competencia social y ciudadana en ciencias sociales al finalizar las etapas de Primaria y Secundaria. Investigación en la Escuela, 78, 19-30.

Monteagudo, J. y Villa, J. L. (2011). La evaluación de las competencias básicas en la materia de Historia en $4 .^{\circ}$ de ESO en la Región de Murcia. En P. Miralles Martínez, S. Molina Puche y A. Santisteban Fernández (Eds.), La evaluación en el proceso de enseñanza y aprendizaje de las ciencias sociales (V. I, pp. 317-325). Murcia: Asociación Universitaria de Profesorado de Didáctica de las Ciencias Sociales.

Monteagudo, J., Miralles, P. y Villa, J. L. (2014). Evaluación de la materia de Historia en Secundaria. El caso de la Región de Murcia. Berlín: Publicia.

Monteagudo, J., Molina, S., y Miralles, P. (2015). Opiniones sobre evaluación de los profesores de segundo ciclo de ESO de Geografía e Historia en España. El caso de la Región de Murcia. Revista Mexicana de Investigación Educativa, 20(66), 737-761.

Monteagudo, J., Villa, J. L., y Miralles, P. (2015). Las prácticas de evaluación en Historia de 4. ${ }^{\circ}$ de ESO según la opinión de los inspectores de Educación. Didáctica de las Ciencias Experimentales y Sociales, 29, 61-88.

Pérez, M. ${ }^{a}$ L. y Carretero M. ${ }^{a}$ R. (2009). La evaluación del aprendizaje en la Educación Secundaria: análisis de un proceso de cambio. Límite. Revista de Filosofía y Psicología, 4(19), 93-126.

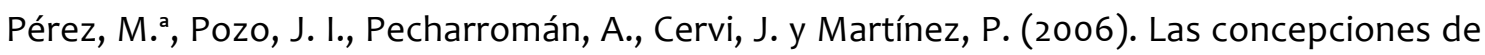
los profesores de educación secundaria sobre el aprendizaje y la enseñanza. En J. I. Pozo, N. Scheuer, M. Pérez Echeverría, M. Mateos, E. Martín y M. De la Cruz, Nuevas 
formas de pensar la enseñanza y el aprendizaje. Las concepciones de profesores y alumnos (pp. 289-304). Barcelona: Graó.

Perkins, D. (1997). La escuela inteligente. Barcelona: GEDISA.

Remesal, A. (2011). Primary and secondary teachers' conceptions of assessment: A qualitative study. Teaching and Teacher Education, 27, 472-482.

Sainz, L. (2002). La evaluación del aprendizaje: Educación vs Autoridad: una propuesta formativa u no impositiva en pos del consenso profesor-estudiante. Revista Electrónica Interuniversitaria de Formación del Profesorado, 5 (1).

Stufflebeam D. y Shinkfield, A. (1995).Evaluación Sistemática. Guía Teórica y Práctica. Madrid: Paidós.

Villa, J. L. y Alonso, J. (1996). ¿Cómo se evalúa el aprendizaje en enseñanzas medias? Revista de Ciencias de la Educación, 168, 473-503.

Zaragoza Raduà, J. M. ${ }^{a}$ (2003). Actitudes del Profesorado de Secundaria Obligatoria hacia la Evaluación de los Aprendizajes de los Alumnos. Tesis doctoral. Barcelona: Universidad Autónoma de Barcelona. 